Comment. Math. Helv. 75 (2000) 457-477

(C) 2000 Birkhäuser Verlag, Basel

$0010-2571 / 00 / 030457-21 \$ 1.50+0.20 / 0$

Commentarii Mathematici Helvetici

\title{
Fibre products, non-positive curvature, and decision problems
}

Gilbert Baumslag, Martin R. Bridson, Charles F. Miller III and Hamish Short

\begin{abstract}
We give a criterion for fibre products to be finitely presented and use it as the basis of a construction that encodes the pathologies of finite group presentations into pairs of groups $P \subset G$ where $G$ is a product of hyperbolic groups and $P$ is a finitely presented subgroup. This enables us to prove that there is a finitely presented subgroup $P$ in a biautomatic group $G$ such that the generalized word problem for $P \subset G$ is unsolvable and $P$ has an unsolvable conjugacy problem. An additional construction shows that there exists a compact non-positively curved polyhedron $X$ such that $\pi_{1} X$ is biautomatic and there is no algorithm to decide isomorphism among the finitely presented subgroups of $\pi_{1} X$.
\end{abstract}

Mathematics Subject Classification (2000). 20F32, 20F05, $20 \mathrm{~F} 10$.

Keywords. Finitely presented groups, fibre products, decision problems, non-positive curvature, hyperbolic groups, automatic groups.

\section{Introduction}

It is rather straightforward to construct finitely generated subgroups with unsolvable decision problems inside apparently well-behaved groups (see [18], [17], [20], [4]); constructing finitely presented subgroups with unsolvable decision problems is a much more delicate matter. In [3] we showed that the finitely presented subgroups of products of hyperbolic groups can be rather complicated by concentrating on the complexity of the word problem: the optimal isoperimetric inequality satisfied by finitely presented subgroups may be significantly worse than the quadratic isoperimetric inequality satisfied by the ambient group. In the present article we turn our attention to the other basic decision problems: the conjugacy problem, the isomorphism problem and the generalized word problem. We show that all of these problems are unsolvable for certain finitely presented subgroups

This work was supported in part by NSF Grants \#9103098 (first author) and \#9401362 (second author), an EPSRC Advanced Fellowship (second author), the Australian Research Council (all authors), and the British Council (second and fourth authors). 
of semihyperbolic groups.

Theorem A. There exists a torsion-free word hyperbolic group $\Gamma$ and a finitely presented subgroup $P \subset \Gamma \times \Gamma$ such that there is no algorithm to decide membership of $P$, and the conjugacy problem for $P$ is unsolvable. (One can arrange for $\Gamma$ to be the fundamental group of a compact negatively curved 2-complex.)

Theorem B. There exists a non-positively curved 4-dimensional cubical complex $K$ with biautomatic fundamental group $G$, and a recursive class of finitely presented subgroups $H_{n} \subset G(n \in \mathbb{N})$ such that there is no algorithm to determine whether or not $H_{n}$ is (abstractly) isomorphic to $H_{1}$.

With regard to Theorem A, note that $\Gamma \times \Gamma$ itself does have a solvable conjugacy problem [12].

Any compact piecewise euclidean complex of dimension $n$ can be isometrically embedded in a closed manifold of dimension $2 n+1$ that has a polyhedral metric of non-positive curvature. The universal cover of a complete non-positively curved space is contractible, and therefore the homotopy type of its covering spaces are determined by the isomorphism classes of the corresponding subgroups. Thus we have:

Corollary C. There exists a closed non-positively curved manifold of dimension 9 and a recursive class of finitely presented subgroups of $\pi_{1} M$ such that there is no algorithm to determine homotopy equivalence between the covering spaces corresponding to these subgroups.

The group $G$ in Theorem B is a direct product $\hat{\Gamma} \times \Gamma$, where $\Gamma$ is a torsion-free word hyperbolic group and $\hat{\Gamma}$ is an HNN-extension of $\Gamma$ given by $\langle\Gamma, \tau| \tau^{-1} a_{1} \tau=$ $\left.a_{1}\right\rangle$ where $a_{1} \in \Gamma$ generates a maximal cyclic subgroup.

We shall make extensive use of the following fibre product construction. Associated to any short exact sequence $1 \rightarrow N \rightarrow \Gamma \rightarrow Q \stackrel{\rho}{\rightarrow} 1$ one has the fibre product $P \subset \Gamma \times \Gamma$ where $P=\left\{\left(\gamma_{1}, \gamma_{2}\right) \mid \rho\left(\gamma_{1}\right)=\rho\left(\gamma_{2}\right)\right\}$. If $Q$ is finitely presented and $\Gamma$ is finitely generated then $P$ is finitely generated. $P$ is essentially the graph of the equality relation $=_{Q}$ in the quotient $Q$, and hence questions about equality in $Q$ translate into questions about membership in $P$.

In the special case when $\Gamma$ is free and $Q$ is a finitely presented group with undecidable word problem, the corresponding $P$ will have a number of undecidable problems (see [18]). In that case, however, $P$ is almost never finitely presented (see [5], [14]). To achieve results concerning undecidable properties of finitely presented subgroups, we exploit an enhanced version of a construction due to Rips. In this construction one gets a short exact sequence with $N$ finitely generated and $\Gamma$ word hyperbolic, and if $Q$ is given by an aspherical presentation, the associated fibre product $P$ is finitely presented. This is implied by the next result. Recall that 
a group is said to be of type $F_{n}$ if it has an Eilenberg-Maclane space with only finitely many $k$-cells for $k \leq n$.

1-2-3 Theorem. Suppose that $1 \rightarrow N \rightarrow \Gamma \stackrel{p}{\rightarrow} Q \rightarrow 1$ is exact, and consider the fibre product:

$$
P:=\left\{\left(\gamma_{1}, \gamma_{2}\right) \mid p\left(\gamma_{1}\right)=p\left(\gamma_{2}\right)\right\} \subset \Gamma \times \Gamma .
$$

If $N$ is finitely generated, $\Gamma$ is finitely presented and $Q$ is of type $F_{3}$, then $P$ is finitely presented.

The name of this theorem comes from the fact that the groups $N, \Gamma$ and $Q$ are assumed to be of type $F_{1}, F_{2}$ and $F_{3}$ respectively.

According to one's taste and background, one might wish to view our results as being about hyperbolic groups, (bi)automatic groups, or spaces of non-positive curvature. These different perspectives are connected by the following facts:

(1) if $X$ is a compact space that is negatively curved in the sense of Alexandrov [6], then $\pi_{1} X$ is torsion-free and hyperbolic in the sense of Gromov [13];

(2) hyperbolic groups are biautomatic [11];

(3) the Cartesian product of two negatively (or non-positively) curved spaces is non-positively curved [6];

(4) the direct product of finitely many biautomatic groups is biautomatic [11];

(5) the fundamental groups of many (but not all) compact non-positively curved complexes are known to be biautomatic (see [12], [16]);

(6) biautomatic groups are semihyperbolic [1];

(7) semihyperbolic groups are bicombable [22].

This paper is organised as follows: In the first section we consider Peiffer sequences and the second homotopy module of a presentation, prior to proving the $1-2-3$ theorem in section 2 .

In section 3 we discuss the enhanced Rips theorem, and use it to prove Theorem A.

In section 4, several results are established about the structure of centralizers in direct products and certain HNN extensions, and these results together with Theorem A are used to prove Theorem B.

In biautomatic groups, most problems concerning centralizers of finitely generated subgroups are algorithmically solvable. In contrast, in section 5 we use results from earlier sections to show that basic questions concerning normalizers in biautomatic groups are algorithmically unsolvable.

The discussions leading to this paper began while the authors were visiting the ANU at Canberra in July 1996, and were continued at the University of Melbourne in August 1996 and the Université de Provence in August 1997. We wish to thank these three universities for their financial support and hospitality. 


\section{$\pi_{2}$ and relations in finitely generated kernels}

In the course of this section and the next we shall prove the 1-2-3 Theorem. It turns out that the key to understanding finite presentability of our fibre products lies with an understanding of the relations that hold among the generators of the kernel in the corresponding short exact sequence $1 \rightarrow N \rightarrow \Gamma \rightarrow Q \rightarrow 1$. The assumption that $Q$ is of type $F_{3}$ plays a central role in this understanding; in order to see why, we need an algebraic reformulation of the condition $F_{3}$.

\subsection{Type $F_{3}$ and $\pi_{2}$}

Let $Y$ be an Eilenberg-Maclane space for $Q$ with a finite 3 -skeleton. Contracting a maximal tree if necessary, we may assume that $Y$ has a single vertex, and thus the 2-skeleton $Y^{(2)}$ corresponds to a finite presentation $\mathcal{P}_{Y}$ of $Q$, with generators given by the 1-cells and relators given by the attaching maps of the 2-cells. Because $Y^{(3)}$ is finite, $\pi_{2} \mathcal{P}_{Y}:=\pi_{2} Y^{(2)}$ is finitely generated as a $Q$-module (the attaching maps of the finitely many 3-cells of $Y$ give a generating set). This finite generation property is useful because it admits the following algebraic interpretation. (Basic references include [15], [7], [2].)

\subsection{Identity sequences and Peiffer moves}

Let $\mathcal{P}=\langle\mathcal{X} \mid \mathcal{R}\rangle$ be a presentation of the group $G$. We consider finite sequences $\sigma=\left(c_{1}, \ldots, c_{m}\right)$ with $c_{i}=w_{i} r_{i} w_{i}^{-1}$ for some $r_{i} \in \mathcal{R}^{ \pm 1}$ and $w_{i}$ in the free monoid $\left(\mathcal{X} \cup \mathcal{X}^{-1}\right)^{*}$, and $\varepsilon= \pm 1$. If the product $\prod c_{i}$ is freely equal to the empty word in $F(\mathcal{X})$, then $\sigma$ is called an identity sequence over $\mathcal{P}$. We consider the equivalence relation on identity sequences generated by the following operations (Peiffer moves).

(P1) Replace some $w_{i}$ by a word freely equal to it.

(P2) Delete or insert consecutive terms where one is identically equal to the (formal) inverse of the other.

(P3) Replace two consecutive terms $\left(c_{i}, c_{i+1}\right)$ by either $\left(c_{i+1}, c_{i+1}^{-1} c_{i} c_{i+1}\right)$ or $\left(c_{i} c_{i+1} c_{i}^{-1}, c_{i}\right)$

Juxtaposition of sequences induces a group structure on the set of equivalence classes; one checks that this is an abelian group. The free group $F(\mathcal{X})$ acts on equivalence classes of identity sequences: $u \cdot\left(c_{1}, \ldots, c_{m}\right)=\left(u c_{1} u^{-1}, \ldots, u c_{m} u^{-1}\right)$. This induces an action of $G$ on the set of equivalence classes. The resulting $G$ module is naturally isomorphic to $\pi_{2} \mathcal{P}$, the second homotopy module of the standard 2-complex associated to the presentation $\mathcal{P}$.

The class of the empty sequence is the neutral element of this group. Thus the condition $\pi_{2} \mathcal{P}=0$ means that any identity sequence can be reduced to the empty sequence by a finite number of Peiffer moves. And the condition that we 
are interested in, $\pi_{2} \mathcal{P}$ being finitely generated as a $G$-module, is equivalent to the statement that there is a finite set of identity sequences such that any identity sequence over $\mathcal{P}$ can be reduced by Peiffer moves to the juxtaposition of finitely many conjugates of sequences from this set.

\subsection{Words as maps}

In what follows it is useful to adopt the viewpoint that words are functions that assign to ordered alphabets $\mathcal{A}$ of a fixed cardinality elements of the free monoid on $\mathcal{A} \cup \mathcal{A}^{-1}$. Thus if $\mathcal{A}=\{a, b\}$ and $\mathcal{A}^{\prime}=\left\{a^{\prime}, b^{\prime}\right\}$, and one defines a word $V$ by $V(\mathcal{A}):=a b a$, then $V\left(\mathcal{A}^{\prime}\right)$ denotes the word $a^{\prime} b^{\prime} a^{\prime}$.

An advantage of this viewpoint is that it provides a language in which to express the fact that if, for instance, the left side of a free equality involves only a proper subset of the generators, then the equality remains valid if one replaces the symbols that appear only on the right side by any other symbols. Thus, if $U_{1}(\mathcal{A})=U_{2}(\mathcal{A}, \mathcal{X})$ freely, then $U_{1}(\mathcal{A})=U_{2}\left(\mathcal{A}, \mathcal{X}^{\prime}\right)$ for any alphabet in 1-1 correspondence with $\mathcal{X}$.

(We shall continue to use the term 'word' in the more traditional sense of an evaluation of $V$ on a specific alphabet when this is unlikely to cause confusion.)

\subsection{Presentations coming from short exact sequences}

Consider a short exact sequence $1 \rightarrow N \rightarrow \Gamma \stackrel{p}{\rightarrow} Q \rightarrow 1$; suppose that $\Gamma$ and $Q$ are finitely presentable and that $N$ is finitely generated. Let $\underline{a}=\left\{a_{1}, \ldots, a_{n}\right\}$ be a finite generating set for $N$, and let $\langle\mathcal{X} \mid \mathcal{R}\rangle$ be a finite presentation for $Q$. We shall define a finite presentation

$$
\Gamma \cong\left\langle\underline{a}, \underline{x} \mid S_{1}, S_{2}, S_{3}\right\rangle,
$$

where the generators $\underline{x}=\left\{x_{1}, \ldots, x_{m}\right\}$ are lifts of $\mathcal{X}$ (so the natural map $F(\mathcal{X}) \rightarrow$ $Q$ factors as $F(\mathcal{X}) \cong F(\underline{x}) \rightarrow \Gamma \rightarrow Q)$.

The first set of relations $S_{1}$ arise from the fact that $N$ is normal. For each $x_{j} \in \underline{x}, a_{i} \in \underline{a}$, and $\varepsilon= \pm 1$ we choose a word $V_{i, j, \varepsilon}$ so that in $\Gamma$ the following words represent the identity:

$$
x_{j}^{\varepsilon} a_{i} x_{j}^{-\varepsilon} V_{i, j, \varepsilon}(\underline{a}) .
$$

The second set of relations $S_{2}$ are obtained by lifting the defining relations of $Q$. For each $r \in R$ we choose a word $U_{r}$ so that the following words represent $1 \in \Gamma$ :

$$
r(\underline{x}) U_{r}(\underline{a}) .
$$

In the quotient of the free group $F(\underline{a}, \underline{x})$ by these two sets of relations, any word $w(\underline{a}, \underline{x})$ is equal to a word of the form $w_{a}(\underline{a}) w_{x}(\underline{x})$. If $w(\underline{a}, \underline{x})=1 \mathrm{in} \Gamma$, then 
$w_{x}(\underline{x})=1$ in $Q$, and hence there is an equality of the form $w_{x}=\prod z_{i} r_{i} z_{i}^{-1}$ (with $r_{i} \in \mathcal{R}^{ \pm 1}$ ) in the free group on $\underline{x}$. Modulo the relations $S_{1}$ and $S_{2}$, this product is equal to a word over $\underline{a}$. It follows that $\Gamma$ is the quotient of $F(\underline{a}, \underline{x})$ by the relations $S_{1}$ and $S_{2}$ together with some additional relations of the form

$$
w(\underline{a})=1
$$

Because $\Gamma$ is assumed to be finitely presented, a finite number of these extra relations suffice; $S_{3}$ is a fixed choice of such a finite sufficient set. Note that in general $\left\langle\underline{a} \mid S_{3}\right\rangle$ is not a presentation of $N$; other (in general infinitely many) consequences of $S_{1}, S_{2}, S_{3}$ are usually required to present $N$.

\subsection{Relations in $N$ coming from identity sequences}

We explain how identity sequences $\sigma=\left(c_{1}, \ldots, c_{m}\right)$ over our fixed presentation $\langle\mathcal{X} \mid \mathcal{R}\rangle$ for $Q$ give rise to relations among the generators $\underline{a}$ of $N$.

If $c_{j}=w_{j}^{-1} r_{j} w_{j}$ then we first associate to $\sigma$ the product

$$
\zeta_{\sigma}:=\prod_{j=1}^{m} w_{j}^{-1}(\underline{x}) r_{j}(\underline{x}) U_{r_{j}}(\underline{a}) w_{j}(\underline{x}),
$$

where the words $U_{r}$ are as in $S_{2}$ (so $r(\underline{x}) U_{r}(\underline{a})=1$ in $\Gamma$ ). Because the word obtained by deleting all occurrences of the letters $\underline{a} \cup \underline{a}^{-1}$ from this word is freely equal to the empty word, we can use relations of type $S_{1}$ to reduce $\zeta_{\sigma}$ to a word involving only the letters $\underline{a}^{ \pm 1}$ : one simply follows a pattern of pinches by which the redacted word is reduced to the empty word; at each stage instead of simply cancelling $x x^{-1}$ one applies relations from $S_{1}$ to replace a subword of the form $x U(\underline{a}) x^{-1}$ by a word $U^{\prime}(\underline{a})$.

Let $Z_{\sigma}(\underline{a}) \in F(\underline{a})$ be the word obtained by this reduction process.

Lemma 1.1. $Z_{\sigma}(\underline{a})=1$ in $N$.

Proof. $\zeta_{\sigma}$ is obviously equal to 1 in $\Gamma$, and $Z_{\sigma}$ is obtained from $\zeta_{\sigma}$ by applying defining relations of $\Gamma$.

We continue to work with the short exact sequence $1 \rightarrow N \rightarrow \Gamma \rightarrow Q \rightarrow 1$ and the presentation of $\Gamma$ defined in (1.4). In addition, we now suppose that $\pi_{2}$ of the finite presentation $\langle\mathcal{X} \mid \mathcal{R}\rangle$ of $Q$ with which we are working is finitely generated as a $Q$-module. We fix a finite set of identity sequences $\sigma_{1}, \ldots, \sigma_{M}$ whose Peiffer equivalence classes form a $Q$-generating set for $\pi_{2}$, and choose words $Z_{\sigma_{i}}$ as above.

The following is the main result of this section (related results concerning presentations and extensions, expressed in the language of pictures, can be found in $[2])$. 
Theorem 1.2. (Relations in $N)$ Let $W$ be a word. $W(\underline{a})=1$ in $\Gamma$ if and only if $W(\underline{a})$ is freely equal $($ in $F(\underline{a}, \underline{x}))$ to a product of conjugates of:

1. $s(\underline{a}, \underline{x})$, where $s \in S_{1} \cup S_{3}$,

2. $Z_{\sigma_{1}}(\underline{a}), \ldots, Z_{\sigma_{M}}(\underline{a})$, and

3. commutators $[s(\underline{a}, \underline{x}), b]$, with $s \in S_{2}^{ \pm 1}, b \in \underline{a}^{ \pm 1}$.

Proof. The "if" direction is obvious, in the light of Lemma 1.1. The point of the theorem, then, is that instead of needing the full force of the relators $S_{2}$ in order to show that $W(\underline{a})=1$ in $\Gamma$ (and hence in $N$ ), one only needs to use the fact that these relators commute with the generators $\underline{a}$.

Let $\widetilde{\Gamma}$ be the quotient of the free group $F(\underline{a}, \underline{x})$ by the stated relations. We must show that if $W(\underline{a})=1$ in $\Gamma$ then $W(\underline{a})=1$ in $\widetilde{\Gamma}$. But if $W(\underline{a})=1$ in $\Gamma$ then modulo relators of type $S_{1}$ and $S_{3}$, it is equal to a product of conjugates of relators of type $S_{2}$, so it suffices to consider the case where $W(\underline{a})=\prod p_{i}^{-1} s_{i} p_{i}$ in the free group $F(\underline{a}, \underline{x})$, where each $s_{i} \in S_{2}$.

This is the point at which we see why it is important to have the relators $[s(\underline{a}, \underline{x}), b]$ : these relations mean that the action of $g p\{\underline{a}\}$ by conjugation on the images in $\widetilde{\Gamma}$ of each $s \in S_{2}$ is trivial, and hence the above product decomposition of $W$ shows that $W(\underline{a})=\prod \bar{p}_{i}^{-1} s_{i} \bar{p}_{i}$ in $\widetilde{\Gamma}$, where $\bar{p}_{i}$ is the word obtained from $p_{i}$ by deleting all occurrences of letters from $\underline{a}$. More precisely, one achieves the desired deletion of the $\underline{a}$ letters by making repeated use of the following calculation:

Take the free identity

$$
a^{-1} v(\underline{x})^{-1} s(\underline{a}, \underline{x}) v(\underline{x}) a=v(\underline{x})^{-1}\left[v(\underline{x}) a v(\underline{x})^{-1}\right]^{-1} s(\underline{a}, \underline{x})\left[v(\underline{x}) a v(\underline{x})^{-1}\right] v(\underline{x})
$$

and use relations of type $S_{1}$ to replace the terms in square brackets by words over $\underline{a}$, then use the relations $[s, b]$ to pass these new words through $s$ and cancel them.

At this stage we have reduced to the problem of showing that any product

$$
W^{\prime}(\underline{a}):=\prod_{i=1}^{m} t_{i}(\underline{x})^{-1} s_{i}(\underline{a}, \underline{x}) t_{i}(\underline{x}), \text { with } s_{i} \in S_{2}
$$

is trivial in $\widetilde{\Gamma}$. It is for this purpose that we need the relators $Z_{i}$. The key observation is that $W^{\prime}(\underline{a})=\zeta_{\sigma}$ (notation of $(*)$ ), where $\sigma$ is the identity sequence $\left(t_{1}^{-1} r_{1} t_{1}, \ldots, t_{m}^{-1} r_{m} t_{m}\right)$, and $s_{i}(\underline{a}, \underline{x})=r_{i}(\underline{x}) U_{r_{i}}(\underline{a})$. The key to showing that $W^{\prime}(\underline{a})=\zeta_{\sigma}=1$ in $\widetilde{\Gamma}$ is:

Lemma 1.3. If $\sigma$ and $\sigma^{\prime}$ are Peiffer equivalent identity sequences over $\langle\mathcal{X} \mid \mathcal{R}\rangle$ then $\zeta_{\sigma}(\underline{a}, \underline{x})=\zeta_{\sigma^{\prime}}(\underline{a}, \underline{x})$ in $\widetilde{\Gamma}$.

Proof. For Peiffer moves of type (P1) and (P2), the claim if obvious. For (P3), we need to show that the products $(*)$ associated to identity sequences $\left(c_{1}, c_{2}\right)$ and 
$\left(c_{1} c_{2} c_{1}^{-1}, c_{1}\right)$, with $c_{i}=w_{i}^{-1} r_{i} w_{i}$, are equal modulo $S_{1}$. Writing $s_{i}=r_{i}(\underline{x}) U_{r_{i}}(\underline{a})$ and $\tau_{i}=w_{i}^{-1}(\underline{x}) s_{i}(\underline{a}, \underline{x}) w_{i}(\underline{x})$, what we must show is:

$$
\begin{gathered}
\tau_{1}(\underline{a}, \underline{x}) \tau_{2}(\underline{a}, \underline{x})= \\
{\left[w_{1}^{-1}(\underline{x}) r_{1}(\underline{x}) w_{1}(\underline{x})\right]\left[w_{2}^{-1}(\underline{x}) s_{2}(\underline{a}, \underline{x}) w_{2}(\underline{x})\right]\left[w_{1}^{-1}(\underline{x}) r_{1}(\underline{x}) w_{1}(\underline{x})\right]^{-1} \tau_{1}(\underline{a}, \underline{x}),}
\end{gathered}
$$

in $\widetilde{\Gamma}$.

If in the square brackets we had $s_{1}(\underline{a}, \underline{x})$ instead of $r_{1}(\underline{x})$, then these expressions would be freely equal. But $r_{1}(\underline{x})$ is simply the word obtained by deleting all occurrences of $\underline{a}$ from $s_{1}(\underline{a}, \underline{x})$, so by applying the argument of the third and fourth paragraphs of the proof to the conjugating element $p=\left[w_{1}^{-1}(\underline{x}) r_{1}(\underline{x}) w_{1}(\underline{x})\right] w_{2}^{-1}(\underline{x})$, we see that the above equality is valid in $\widetilde{\Gamma}$.

Returning to the proof of the theorem, since any identity sequence $\sigma$ over $\langle\mathcal{X} \mid \mathcal{R}\rangle$ is Peiffer equivalent to a product of conjugates of the generating sequences $\sigma_{i}$, we have that $\zeta_{\sigma}$ is equal to a product of conjugates of the $\zeta_{\sigma_{i}}$ in $\widetilde{\Gamma}$. But $\zeta_{\sigma_{i}}=Z_{i}$ in $\widetilde{\Gamma}$, and we have included the relations $Z_{i}=1$ in the defining relations of $\widetilde{\Gamma}$.

\section{The 1-2-3 Theorem}

Associated to the short exact sequence $1 \rightarrow N \rightarrow \Gamma \stackrel{p}{\rightarrow} Q \rightarrow 1$ one has the fibre product:

$$
P:=\left\{\left(\gamma_{1}, \gamma_{2}\right) \mid p\left(\gamma_{1}\right)=p\left(\gamma_{2}\right)\right\} \subset \Gamma \times \Gamma .
$$

We are concerned with the finite presentability of $P$.

We maintain the notations of the previous section; these include $\langle\mathcal{X} \mid \mathcal{R}\rangle$, a fixed finite presentation of $Q$ for which $\pi_{2}$ is finitely generated as a $Q$-module; $\underline{a}$ and $\underline{x}$, the generators of $\Gamma$; the relations $S_{1}, S_{2}$ and $S_{3}$ of $\Gamma$; and the words $Z_{\sigma_{i}}(\underline{a})$ considered in Lemma 1.1.

The following is easily verified:

Lemma 2.1. $P$ is generated by

1. $\underline{X}=\{X=(x, x) \mid x \in \underline{x}\}$,

2. $\underline{a}^{L}=\left\{a^{L}=(a, 1) \mid a \in \underline{a}\right\}$, and

3. $\underline{\bar{a}}^{R}=\left\{a^{R}=(1, a) \mid a \in \underline{a}\right\}$.

We add to the above list superfluous generators $\underline{A}:=\left\{A=a^{L} a^{R} \mid a \in \underline{a}\right\}$.

The following is a more precise statement of the 1-2-3 Theorem.

Theorem 2.2. $P$ has finite presentation $\left\langle\underline{X} \cup \underline{A} \cup \underline{a}^{L} \cup \underline{a}^{R} \mid \mathcal{R}\right\rangle$, where $\mathcal{R}$ consists of:

1. $A^{-1}\left(a^{L} a^{R}\right)$ and $\left[a^{R}, a^{L}\right]$ for all $a \in \underline{a}$, 
2. $s(\underline{A}, \underline{X})$ for all $s \in S_{1} \cup S_{2}$,

3. $s\left(\underline{a}^{L}\right)$ and $s\left(\underline{a}^{R}\right)$ for all $s \in S_{3}$,

4. $Z_{\sigma_{i}}\left(\underline{a}^{L}\right)$ and $Z_{\sigma_{i}}\left(\underline{a}^{R}\right)$ for $i=1, \ldots, M$,

5. $s\left(\underline{a}^{L}, \underline{X}\right)$ and $s\left(\underline{a}^{R}, \underline{X}\right)$ for all $s \in S_{1}$, and

6. the commutators $\left[s\left(\underline{a}^{L}, \underline{X}\right), b^{L}\right]$ and $\left[s\left(\underline{a}^{R}, \underline{X}\right), b^{R}\right]$, with $s \in S_{2}, b \in \underline{a}$.

In fact the relators (6) can be deduced from (1), (2), and (5); we include them as they play a crucial role in our proof.

Proof. It is easily verified that these relations are valid in $\Gamma \times \Gamma$ and hence $P$. The relations of type (1) are rather obvious. The relations of type (2), together with the relations $s(\underline{A}), s \in S_{3}$ that one deduces from (1) and (3), are the defining relations of the diagonal of $\Gamma \times \Gamma$ (which is contained in $P$ ). Relations of type (3) and (4) come from Theorem 1.2, as do the relations of type (6). Actually, in order to see that (6) really corresponds to the relations stated in Theorem 1.2 one needs the relations in (5), which encode the fact that the action by conjugation of $X \in \underline{X}$ on $\underline{a}^{L}$ (resp. $\underline{a}^{R}$ ) is the same as the action of $(\underline{x}, 1)($ resp. $(1, \underline{x})$ ).

It remains to show that if a word $W$ in the free group on $\underline{X} \cup \underline{A} \cup \underline{a}^{L} \cup \underline{a}^{R}$ represents the identity in $P$, then it is a consequence of the stated relations.

Case 1: If $W$ consists only of letters from one of $\underline{a}^{L}$ or $\underline{a}^{R}$, then Theorem 1.2 enables us to express $W$ as a product of conjugates of relations of type (3), (4), (5) and (6).

Case 2: If $W$ consists only of letters from $\underline{A} \cup \underline{a}^{L} \cup \underline{a}^{R}$, then modulo relations of type (1), $W$ is equal to a word of the form $w_{L}\left(\underline{a}^{L}\right) w_{R}\left(\underline{a}^{R}\right)$. If $W=1$ in $P$ then $w_{L}\left(\underline{a}^{L}\right)=w_{R}\left(\underline{a}^{R}\right)=1$ in $P$, and we are back in Case 1 .

Case 3: In the general case, one can replace all occurrences of the letters $A \in \underline{A}$ by $a^{L} a^{R}$ using (1), and then use the relators of type (5) to replace $W$ by a word $W^{\prime}$ of the form $W_{1}(\underline{X}) W_{2}\left(\underline{a}^{L}, \underline{a}^{R}\right)$. Projecting to the right-hand factor of $\Gamma \times \Gamma$ and then to $Q$, one sees that $W_{1}(\mathcal{X})=1$ in $Q$, and hence $W_{1}(\underline{X})$ is freely equal to a product of the form $\prod p_{i}^{-1}(\underline{X}) r_{i}(\underline{X}) p_{i}(\underline{X})$, with $r_{i} \in \mathcal{R}$. Modulo relations of type (2), this product is equal to a word in the generators $\underline{A}$. Thus we have reduced to Case 2 .

\section{The proof of Theorem A}

As we noted in the introduction, this is the second article in which the authors have sought to demonstrate the diverse nature of finitely presented subgroups in products of hyperbolic groups by examining the nature of their decision problems. In the case of the word problem [3] our discussion focused on the complexity of solutions because existence was a trivial matter (any finitely presented subgroup of a group with a solvable word problem obviously has a solvable word problem). In contrast, solvability of the conjugacy problem is not inherited by finitely pre- 
sented subgroups in general (see [18] — for an example of such a finite index subgroup see [8]). Thus, although products of biautomatic and related groups have a solvable conjugacy problem [11], it is possible that their finitely presented subgroups may not. This opens up the possibility of using the (un)solvability of the conjugacy problem as an invariant for identifying finitely presented subgroups of various classes of biautomatic groups that are not themselves biautomatic (or more generally bicombable [1], [21]).

Rips [20] associates to any finite presentation of a group $Q$ a short exact sequence $1 \rightarrow N \rightarrow \Gamma \rightarrow Q \rightarrow 1$, where $\Gamma$ is hyperbolic and $N$ is finitely generated. By performing the Rips construction more carefully, one can arrange for $\Gamma$ to be the fundamental group of a compact negatively curved 2-complex in which the 2-cells are regular right-angled hyperbolic pentagons. This modified Rips construction, described in [6] and [23], yields the following:

Theorem 3.1. (Enhanced Rips Construction) There is an algorithm that associates to any finite group presentation $\mathcal{Q}$ a compact, negatively curved, piecewise hyperbolic 2-dimensional complex $K$ and a short exact sequence

$$
1 \rightarrow N \rightarrow \Gamma \rightarrow Q \rightarrow 1
$$

such that

1. $Q$ is the group given by the presentation $\mathcal{Q}$,

2. $K$ has a single vertex $x_{0}$,

3. the 2-cells of $K$ are right-angled hyperbolic pentagons (each side of which crosses several 1-cells),

4. $\Gamma=\pi_{1}\left(K, x_{0}\right)$,

5. $N$ has a finite generating set $\underline{a}$, of cardinality at least 2 ,

6. each of the 1-cells in $K$ is the unique closed geodesic in its homotopy class,

7. the homotopy class of each $a \in \underline{a}$ is represented by one of the 1-cells of $K$,

8. $\Gamma$ is torsion-free,

9. each $a \in \underline{a}$ generates its centralizer.

The first seven items of this theorem are immediate from the constructions in [23] and [6]. Item (8) is a special case of the fact that the fundamental group of any compact non-positively curved space is torsion-free (see [6]). Because $K$ is strictly negatively curved, $\Gamma$ is hyperbolic in the sense of Gromov. Since $\Gamma$ is torsion-free, it follows that the centralizer of every non-trivial element in $\Gamma$ is cyclic. If $a \in \underline{a}$ were a proper power then the homotopy class that it represents would not be represented by a simple closed geodesic, thus (9) follows from (7).

Corollary 3.2. Let $K, \Gamma$ and $\underline{a}$ be as above and let $a \in \underline{a}$. Then $\widehat{\Gamma}=\langle\Gamma, t|$ $\left.t^{-1} a t=a\right\rangle$ is the fundamental group of a compact non-positively curved squared complex, and hence it is biautomatic.

Proof. First we re-metrize $K$ as a squared complex by subdividing each of its 
pentagonal faces into five hyperbolic quadrilaterals by introducing a new vertex in the middle of each face and a new vertex in the middle of each side. We then replace each of these hyperbolic quadrilaterals with a Euclidean square without changing the length of the perimeter of the pentagon. The resulting piecewise Euclidean squared complex is still non-positively curved and the original 1-cells are still closed geodesics. We further subdivide each of the squares into smaller squares whose sides have length half that of the original 1-cells in $K$. We now attach to this remetrized version of $K$ an annulus which is the union of two Euclidean squares: the boundary circles of the annulus are each attached by an isometry to the loop (original 1-cell) representing $a \in \underline{a}$. The resulting 2-complex is again a non-positively curved squared 2-complex (see [6, II.11]), and if the orientations are chosen correctly then the fundamental group is $\widehat{\Gamma}$. By [12] or [16], $\hat{\Gamma}$ is biautomatic.

Theorem A is an immediate consequence of the following:

Theorem $\mathbf{A}^{\prime}$. There exists a compact negatively curved 2-complex $K$ and a finitely presented subgroup $P \subset \pi_{1}(K \times K)=\Gamma \times \Gamma$ such that:

1. the membership problem for $P$ is unsolvable;

2. $P$ has an unsolvable conjugacy problem.

Proof. As input for this theorem we need the fact that there exist groups of type $F_{3}$ with unsolvable word problem. Collins and Miller [9] have proved something considerably stronger: there exist groups $Q$ with a finite 2-dimensional $K(Q, 1)$ and unsolvable word problem. By applying the construction of Theorem 3.1 to such examples we obtain a short exact sequence

$$
1 \rightarrow N \rightarrow \pi_{1} K=\Gamma \stackrel{p}{\rightarrow} Q \rightarrow 1,
$$

where $K$ is a compact, negatively curved 2-complex and $N$ is generated by the finite set $\underline{a}$. Let $X=K \times K$.

We choose generators $\left\{x_{1}, \ldots, x_{n}, a_{1}, \ldots, a_{M}\right\}$ for $\pi_{1} K$, where $\underline{a}=\left\{a_{1}, \ldots, a_{M}\right\}$ and, via $p$, the $\underline{x}=\left\{x_{1}, \ldots, x_{n}\right\}$ are generators for $Q$, as in Theorem 3.1. Take the associated generators $\left(x_{i}, 1\right),\left(1, x_{i}\right),\left(a_{j}, 1\right),\left(1, a_{j}\right)$ for $\pi_{1} X$. According to the 1-2-3 Theorem, the subgroup $P=\left\{\left(\gamma_{1}, \gamma_{2}\right) \mid p\left(\gamma_{1}\right)=p\left(\gamma_{2}\right)\right\} \subset \pi_{1} X$ is finitely presented. Given a word $w$ in the generators $\left(x_{i}, 1\right)$, we ask if this word defines an element of $P$. The answer is "yes" if and only if the same word in the generators $x_{i}$ represents the identity in $Q$. Thus membership of $P$ is undecidable, and (1) is proved.

We use the standard notation $C_{G}(g)$ for the centralizer of the element $g$ in the group $G$. Because $K$ is a negatively curved complex, the centralizer of every $\gamma \in \pi_{1} K$ is cyclic. By Theorem 3.1(9), the centralizer of $\alpha:=\left(a_{i}, a_{i}\right)$ in $\pi_{1} X$ is simply $g p\left\{\left(1, a_{i}\right),\left(a_{i}, 1\right)\right\}$, which is contained in $P$. Thus $(2)$ follows from (1) and the following rather general observation, applied with $H=N \times N \subset P$. 
Lemma 3.3. Let $H \subset P \subset G$ be finitely generated groups. Suppose that $H$ is normal in $G$ and that there exists $a \in H$ such that $C_{G}(a) \subset P$. If there is no algorithm to decide membership of $P$, then $P$ has an unsolvable conjugacy problem.

Proof. We fix finite sets of generators $\mathcal{B}$ for $G$ and $\mathcal{A}$ for $H$. For each $b \in \mathcal{B}$ and $\varepsilon= \pm 1$, let $u_{b, \epsilon}$ be a word in the generators $\mathcal{A}$ so that $b^{\varepsilon} a b^{-\varepsilon}=u_{b, \epsilon}$ in $H$.

Given an arbitrary word $w$ in the generators $\mathcal{B}$, we can use the relations $b^{\varepsilon} a b^{-\varepsilon}=u_{b, \epsilon}$ to rewrite $w a w^{-1}$ as a word $w^{\prime}$ in the generators $\mathcal{A}$. (The length of $w^{\prime}$ is bounded by an exponential function of the length of $w$ and the process of passing from $w$ to $w^{\prime}$ is entirely algorithmic.)

Now ask if $w^{\prime}$ is conjugate to $a$ in $P$. Well, if there exists $h \in P$ such that $h^{-1} a h=w^{\prime}$ then $h w \in C_{G}(a) \subset P$, whence $w \in P$. Thus $w^{\prime}$ is conjugate to $a$ in $P$ if and only if $w \in P$. But there is no algorithm to decide membership of $P$.

We note another consequence of Lemma 3.3 (cfr. [20]):

Proposition 3.4. There exist compact negatively curved 2-complexes $K$ and finitely generated normal subgroups $N \subset \pi_{1} K$ such that the conjugacy problem for $N$ is unsolvable.

Proof. Apply Theorem 3.1 to a group $Q$ with an unsolvable word problem. There is no algorithm to decide if a word $w$ in the generators of $\pi_{1} K$ lies in the normal subgroup $N$ (since membership of $N$ is equivalent to deciding if $w=1$ in $Q$ ). The centralizer of each $a_{i}$ (notation of Theorem 3.1) lies in $N$, so Lemma 3.3 implies that $N$ has an unsolvable conjugacy problem.

\section{The Isomorphism Problem}

Whenever one is trying to prove that the isomorphism problem is undecidable in a certain class of groups, one is invariably faced with the difficulty of ruling out 'accidental' isomorphisms - one needs invariants that allow one to deduce that if some obvious map is not an isomorphism then the groups in question are not isomorphic. The invariants that we shall use in proving Theorem B involve abelian subgroups and centralizers.

Throughout this section, $N, \Gamma, Q=\Gamma / N$ and $P$ will be as in the $1-2-3$ Theorem. (Further restrictions will be imposed on them as we go along.)

Lemma 4.1. Let $H$ be a group. For every $a, h \in H$,

$$
\left\langle H, t \mid t^{-1} a t=h^{-1} a h\right\rangle \cong\left\langle H, \tau \mid \tau^{-1} a \tau=a\right\rangle .
$$

Proof. The desired isomorphism sends $H$ to itself by the identity and sends $t$ to $\tau h$. The inverse sends $\tau$ to $t h^{-1}$. 
We need a bit of technical notation. Take the finite presentation of $\Gamma$ constructed in 1.4. The relations $\left(S_{1}\right)$ imply that for each generator $x_{j}^{\varepsilon}$ of $Q$ and $a_{i}$ of $N$ we have $x_{j}^{\varepsilon} a_{i} x_{j}^{-\varepsilon}=V_{i, j, \varepsilon}$ in $\Gamma$ where each $V_{i, j, \varepsilon}$ is a specific word in $\underline{a}$. These words describe the action on $N$ of any word $w$ in the generators $\underline{x}$ of $Q$. As in the proof of Lemma 3.3, we can use these relations to rewrite $w a_{i} w^{-1}$ as a word $\phi_{w}\left(a_{i}\right)$ in the generators $\underline{a}$ of $N$. (Note that $\phi_{w}\left(a_{i}\right)$ is generally a very complicated word which is not freely conjugate to $a_{i}$.) Using this notation we define the HNN-extensions

$$
\hat{\Gamma}_{w}:=\left\langle\Gamma, t_{w} \mid t_{w}^{-1} a_{1} t_{w}=\phi_{w}\left(a_{1}\right)\right\rangle .
$$

According to Lemma 4.1, the map $\hat{\Gamma}_{w} \rightarrow \hat{\Gamma}_{1}$ that sends $t_{w}$ to $t_{1} w^{-1}$ and restricts to the identity on $N$ is an isomorphism. Extending this map by the identity on the second factor we obtain an isomorphism $\Phi_{w}: \hat{\Gamma}_{w} \times \Gamma \rightarrow \hat{\Gamma}_{1} \times \Gamma$.

Let $\hat{P}_{w}$ be the subgroup of $\hat{\Gamma}_{w} \times \Gamma$ generated by $P \subset \Gamma \times \Gamma$ and $\left(t_{w}, 1\right)$. We shall prove that each of the groups $\hat{P}_{w}$ is finitely presented. We shall then prove that if $\Gamma$ is as in Theorem 3.1 and $Q$ has unsolvable word problem, then there is no algorithm to decide which of the subgroups $\Phi_{w}\left(\hat{P}_{w}\right) \subset \hat{\Gamma}_{1} \times \Gamma$ are isomorphic. This will prove Theorem B, in the light of Corollary 3.2.

First we prove that $\hat{P}_{w}$ is finitely presented.

Lemma 4.2. The group $\hat{P}_{w}$ is finitely presented as an $H N N$-extension by:

$$
\left\langle P, \tau \mid \tau^{-1} a_{1}^{L} \tau=\phi_{w}\left(a_{1}^{L}\right), \tau^{-1} b^{R} \tau=b^{R}, b \in \underline{a}\right\rangle .
$$

Proof. We already know a finite presentation for $P$ given by Theorem 2.2 . We work with that presentation augmented by $\tau$ and the relations displayed above. It is clear that, putting $\tau=\left(t_{w}, 1\right)$, all of the displayed relations involving $\tau$ hold in $\hat{P}_{w}$; what we must show is that the stated relations are sufficient to present $\hat{P}_{w}$.

Suppose that $u$ is a word on the given generators of $\hat{P}_{w}$ which is equal to the identity. If $u$ does not involve $\tau$, then $u$ is a consequence of the relations of $P$ as desired. Thus it suffices to show that if $u$ does involve $\tau$, one can reduce the number of occurences of $\tau$ by applying the stated relations.

$\hat{\Gamma}_{w} \times \Gamma$ can be presented as an HNN-extension of $\Gamma \times \Gamma$ by:

$$
\left\langle\Gamma \times \Gamma, \tau \mid \tau^{-1}\left(a_{1}, 1\right) \tau=\left(\phi_{w}\left(a_{1}\right), 1\right), \tau^{-1}(1, g) \tau=(1, g), g \in \underline{a} \cup \underline{x}\right\rangle
$$

of $\Gamma \times \Gamma$. Applying Britton's Lemma to this relative presentation, we see that $u$ must contain a subword $\tau^{-\varepsilon} v \tau^{\varepsilon}$ where either $\varepsilon=1$ and $v \in\left\langle a_{1}\right\rangle \times \Gamma$ or $\varepsilon=-1$ and $v \in\left\langle\phi_{w}\left(a_{1}\right)\right\rangle \times \Gamma$. In either case $v \in N \times \Gamma$.

Now since $v$ is an element of $P$, we may use the relations of types (1) and (5) from Theorem 3.2 to express $v$ in the form $v=v_{1}\left(\underline{a}^{L}\right) v_{2}\left(\underline{a}^{R}\right) v_{3}(\underline{X})$. Since $v \in N \times \Gamma$, it follows that $v_{3}(\underline{x}) \in N$ and so using relations of type (2) we deduce that $v_{3}(\underline{X})$ is equal in $P$ to a word of the form $v_{4}(\underline{A})$. Then, using relations of 
type (1), we deduce that $v=v_{4}\left(\underline{a}^{L}\right) v_{4}\left(\underline{a}^{R}\right)$. Thus in the case $\varepsilon=1$ we can use the relations of $P$ to deduce $v_{4}\left(\underline{a}^{L}\right)=\left(a_{1}^{L}\right)^{m}$ for some integer $m$, which allows us to apply the listed $\tau$ relations to reduce the number of $\tau$ symbols in $u$. Likewise, in the case $\varepsilon=-1$ we deduce $v_{4}\left(\underline{a}^{L}\right)=\phi_{w}\left(a_{1}^{L}\right)^{m}$ and then apply the listed $\tau$ relations. In either case we reduce the number of $\tau$ symbols in $u$. This completes the proof.

Theorem B is an immediate consequence of the following result.

Theorem 4.3. Let $Q$ be a group of type $F_{3}$ with an unsolvable word problem. Let $\Gamma$ be as in Theorem 3.1 and let $\hat{\Gamma}_{1}$ and $\hat{P}_{w}$ be constructed as above. Then:

(i) $\hat{\Gamma}_{1} \times \Gamma$ is the fundamental group of a non-positively curved cubical complex and hence is bi-automatic;

(ii) there exist explicit finite presentations of $\hat{P}_{w}$ and explicit embeddings $\Phi_{w}$ : $\hat{P}_{w} \hookrightarrow \hat{\Gamma}_{1} \times \Gamma ;$

(iii) $\hat{P}_{w} \cong \hat{P}_{1}$ if and only if $w=1$ in $Q$, and therefore the problem of deciding whether or not $\hat{P}_{w}$ is isomorphic to $\hat{P}_{1}$ is recursively undecidable.

If $w=1$ in $Q$, then the restriction of $\Phi_{w}$ to $\hat{P}_{w}$ sends it isomorphically onto $\hat{P}_{1}$. (The inverse map is the identity on $P$ and sends $\left(t_{1}, 1\right)$ to $\left(t_{w} w, 1\right)$.)

In the light of our previous results, what remains to be proved is that if $w \neq 1$ in $Q$ then $\hat{P}_{w} \neq \hat{P}_{1}$. The way in which we shall distinguish between these groups is by analysing the structures of centralizers of elements. As a first step towards doing so, we need a technical lemma.

Lemma 4.4. Let $G$ be a group, and let $a \in G$ be an element of infinite order such that $\gamma^{-1} a^{m} \gamma=a^{n}$ implies that $\gamma \in\langle a\rangle$ and $m=n$. Let $\widehat{G}$ be the HNN extension $\left\langle G, t \mid t^{-1} a t=a\right\rangle$.

If $g \in \widehat{G}$ is non-trivial, then either

1. $g$ is conjugate to an element of $G$ but $g$ is not conjugate to any element of the subgroup $\langle a, t\rangle$, and in this case $C_{\widehat{G}}(g)=C_{G}(g)$; or

2. $g$ is not conjugate to an element of $G$, nor is it conjugate to any element of the subgroup $\langle a, t\rangle$, in which case the centralizer $C_{\widehat{G}}(g)$ is isomorphic to $\mathbb{Z}$; or

3. $g$ is conjugate to an element of the subgroup $\langle a, t\rangle$, in which case the centralizer $C_{\widehat{G}}(g)$ is conjugate to $\langle a, t\rangle \cong \mathbb{Z}^{2}$.

Proof. $\widehat{G}$ is the fundamental group of a graph of groups with a single vertex at which the vertex group is $G$. There is a single edge, with an infinite cyclic edge group identified with $\langle a\rangle$ at each end; the corresponding stable letter is $t$ and $t^{-1} a t=a$. We now apply the subgroup theory for graphs of groups to the subgroup $C=C_{\widehat{G}}(g)$ of $\widehat{G}$.

Thus $C$ is the fundamental group of a minimal graph of groups whose vertex groups are conjugate to subgroups of $G$ and whose edge groups are conjugate to 
subgroups of $\langle a\rangle$.

There are three cases to consider, corresponding to the three cases of the statement of the lemma:

Case (i): the graph has no edges;

Case (ii): the edge groups are all trivial;

Case (iii): there is a non-trivial edge group.

(i) If there are no edges, then (after conjugation) $C=C \cap G$, and $C \cong C_{G}(g)$.

(ii) In this case $C$ must be infinite cyclic. Otherwise, $C$ would be a non-trivial free product, which the centrality of $g$ renders impossible.

(iii) This is the case $C \neq C \cap G \neq 1$ (after conjugation). We show that if two non-trivial elements $\gamma \in C \cap G$ and $v \in C \backslash G$ commute, then $\gamma$ is a power of $a$ and $v$ is in the subgroup $\langle a, t\rangle$. Write $v$ as a $t$-reduced word $v_{0} t^{\varepsilon_{1}} v_{1} \cdots t^{\varepsilon_{n}} v_{n}$ where $v_{i} \in G$, and $v_{i} \notin\langle a\rangle$ for $i>0$.

Applying Britton's Lemma to the relation

$$
1=v^{-1} \gamma v \gamma^{-1}=v_{n}^{-1} t^{-\varepsilon_{n}} \cdots v_{1}^{-1} t^{-\varepsilon_{1}} v_{0}^{-1} \gamma v_{0} t^{\varepsilon_{1}} v_{1} \cdots t^{\varepsilon_{n}} v_{n} \gamma^{-1}
$$

it follows that $v_{0}^{-1} \gamma v_{0}=a^{m}$ for some $m \in \mathbb{Z}$. If $v_{1} \neq 1$ then $v_{1}^{-1} a_{1}^{m} v_{1}=a_{1}^{k}$, which by the conditions of the Lemma, contradicts the assumption that $v$ is $t$-reduced. So $v=v_{0} t^{\varepsilon_{1}}$.

By the above, conjugating $\gamma$ by $v_{0}$, we get $1=[g, \gamma]^{v_{0}}=\left[g^{v_{0}}, a^{m}\right]$, so that $g^{v_{0}} \in C\left(a^{m}\right)=\langle a, t\rangle$.

Thus $g$ is conjugate to an element $g^{v_{0}}$ of $\langle a, t\rangle$ and hence $C(g)=\langle a, t\rangle$.

In the following lemma $\Gamma$ is a torsion-free hyperbolic group as constructed in Theorem 3.1 and $Q=\Gamma / N$. The element $a_{1} \in N$ satisfies the hypotheses of the previous lemma. For each word $w$ in the generators of $Q$ we have the subgroup $\hat{P}_{w} \subseteq \hat{\Gamma}_{w} \times \Gamma$ generated by $P$ and $\left(t_{w}, 1\right)$. Our goal now is to calculate the centralizers of elements in $\hat{P}_{w}$. In the course of the proof we shall need the observation that the centralizer of $N \subset \Gamma$ is trivial. (Indeed the centralizer of any non-cyclic subgroup in a torsion-free hyperbolic group is trivial.)

Lemma 4.5. The centralizer in $\hat{P}_{w}$ of $h=\left(u_{1}, u_{2}\right) \in \hat{P}_{w}$ can be described as follows:

1. Case (1), $u_{1} \neq 1$ and $u_{2} \neq 1: C_{\hat{P}_{w}}(h)$ is isomorphic to a subgroup of $\mathbb{Z}^{3}$.

2. Case (2), $u_{1}=1$ and $u_{2} \neq 1: C_{\hat{P}_{w}}(h)$ contains $\left(\left(\hat{\Gamma}_{w} \times 1\right) \cap \hat{P}_{w}\right) \times \mathbb{Z}$ as a subgroup of finite index.

3. Case (3), $u_{1} \neq 1$ and $u_{2}=1$ :

(a) If $u_{1}$ is not conjugate in $\hat{\Gamma}_{w}$ to an element of $\left\langle a_{1}, t_{w} w^{-1}\right\rangle$, then $C_{\hat{P}_{w}}(h)$ is isomorphic to a subgroup of $\mathbb{Z} \times \Gamma$.

(b) If $u_{1}$ is conjugate in $\hat{\Gamma}_{w}$ to an element of $\left\langle a_{1}, t_{w} w^{-1}\right\rangle$, then there is a short exact sequence

$$
1 \rightarrow N \rightarrow C_{\hat{P}_{w}}(h) \rightarrow \mathbb{Z}^{2} \rightarrow 1
$$


If $w \in N$, then this sequence splits and $C_{\hat{P}_{w}}(h) \cong \mathbb{Z}^{2} \times N$. If $w \notin N$, then the centre of $C_{\hat{P}_{w}}(h)$ is cyclic.

Proof. Recall that in any direct product $C_{G_{1} \times G_{2}}\left(\left(u_{1}, u_{2}\right)\right)=C_{G_{1}}\left(u_{1}\right) \times C_{G_{2}}\left(u_{2}\right)$. Assume $H \subseteq G_{1} \times G_{2}$ is a subgroup. If $\left(u_{1}, u_{2}\right) \in H$ then $C_{H}\left(\left(u_{1}, u_{2}\right)\right)=$ $H \cap C_{G_{1} \times G_{2}}\left(\left(u_{1}, u_{2}\right)\right)$. Also, projecting onto the second factor $G_{2}$, we obtain an exact sequence

$$
1 \rightarrow H \cap\left(C_{G_{1}}\left(u_{1}\right) \times 1\right) \rightarrow C_{H}\left(\left(u_{1}, u_{2}\right)\right) \stackrel{p_{2}}{\rightarrow} C_{G_{2}}\left(u_{2}\right) .
$$

If $1 \times C_{\Gamma_{2}}\left(u_{2}\right) \subseteq H$ then

$$
\left.H \cap C_{\Gamma_{1} \times \Gamma_{2}}\left(\left(u_{1}, u_{2}\right)\right)=\left[H \cap\left(1 \times C_{\Gamma_{1}}\left(u_{1}\right)\right)\right)\right] \times C_{\Gamma_{2}}\left(u_{2}\right) .
$$

Case (1), $u_{1} \neq 1$ and $u_{2} \neq 1$ : As $\Gamma$ is torsion-free hyperbolic, $C_{\Gamma}\left(u_{2}\right)$ is infinite cyclic. And according to Lemma $4.4, C_{\hat{\Gamma}_{w}}\left(u_{1}\right)$ is free abelian of rank 1 or 2 . Thus $C_{\hat{P}_{w}}(h)=C_{\hat{\Gamma}_{w}}\left(u_{1}\right) \times C_{\Gamma}\left(u_{2}\right)$ is isomorphic to a subgroup of $\mathbb{Z}^{3}$.

Case (2), $u_{1}=1$ and $u_{2} \neq 1$ : In this case $u_{2} \in N$, since $\left(1, u_{2}\right) \in \hat{P}_{w} \cap(1 \times \Gamma)$. And $C_{\Gamma}\left(u_{2}\right)$ is infinite cyclic, so from (\#) we have

$$
1 \rightarrow \hat{P}_{w} \cap\left(C_{\Gamma_{w}}\left(u_{1}\right) \times 1\right) \rightarrow C_{\hat{P}_{w}}(h) \stackrel{p_{2}}{\rightarrow} \mathbb{Z} \rightarrow 1 .
$$

$\left\langle u_{2}\right\rangle$ has finite index in the quotient $\mathbb{Z}$, so $\left.p_{2}^{-1}\left\langle u_{2}\right\rangle=\left[\hat{P}_{w} \cap\left(C_{\Gamma_{w}}\left(u_{1}\right)\right] \times 1\right)\right) \times\left\langle u_{2}\right\rangle$ has finite index in $C_{\hat{P}_{w}}(h)$.

Case (3), $u_{1} \neq 1$ and $u_{2}=1$ : First assume that $u_{1}$ is not conjugate in $\hat{\Gamma}_{w}$ to an element of $\left\langle a_{1}, t_{w} w^{-1}\right\rangle$. Then, by Lemma 4.4, $C_{\hat{\Gamma}_{w}}\left(u_{1}\right)$ is infinite cyclic, say with generator $v \in \hat{\Gamma}_{w}$ where $u_{1}=v^{m}$. In this case $C_{\hat{\Gamma}_{w} \times \Gamma}(h)=\langle v\rangle \times \Gamma$, and hence $C_{\hat{P}_{w}}(h)$ is isomorphic to a subgroup of $\mathbb{Z} \times \Gamma$.

Now assume that there is a word $v(\underline{a}, \underline{x}, t)$ which conjugates $u_{1}$ (in $\left.\hat{\Gamma}_{w}\right)$ to a non-trivial element of $\left\langle a_{1}, t_{w} w^{-1}\right\rangle$. Then $v(\underline{A}, \underline{X}, \tau) \in \hat{P}_{w}$ conjugates $\left(u_{1}, 1\right)$ into $\left\langle\left(a_{1}, 1\right),\left(t_{w} w^{-1}, 1\right)\right\rangle$. Thus it suffices to determine the centralizer in $\hat{P}_{w}$ of an element of the form $h=\left(a_{1}^{d}\left(t_{w} w^{-1}\right)^{e}, 1\right)$. As in (\#), projecting onto the $\hat{\Gamma}_{w}$ factor of $\hat{\Gamma}_{w} \times \Gamma$ we get an exact sequence

$$
1 \rightarrow 1 \times N \rightarrow C_{\hat{P}_{w}}(h) \stackrel{p_{1}}{\rightarrow} C_{\hat{\Gamma}_{w}}\left(a_{1}^{d}\left(t_{w} w^{-1}\right)^{e}\right) .
$$

By Lemma 4.4, $C_{\hat{\Gamma}_{w}}\left(a_{1}^{d}\left(t_{w} w^{-1}\right)^{e}\right)$ is free abelian with generators $a_{1}$ and $t_{w} w^{-1}$. Therefore, since $\left(a_{1}, 1\right)$ and $\left(t_{w} w^{-1}, w^{-1}\right)$ lie in $C_{\hat{P}_{w}}(h)$, the map $p_{1}$ is surjective. If $w \in N$, then we can split $p_{1}$ by sending $a_{1}$ to $\left(a_{1}, 1\right)$ and $t_{w} w^{-1}$ to $\left(t_{w} w^{-1}, 1\right)$. Thus, in this case, $C_{\hat{P}_{w}}(h) \cong N \times \mathbb{Z}^{2}$. 
Because the centre $Z(N)$ of $N$ is trivial, the restriction of $p_{1}$ to $Z\left(C_{\hat{P}_{w}}(h)\right)$ is injective. It follows that either $Z\left(C_{\hat{P}_{w}}(h)\right)$ is cyclic, or else some element of $p_{1}^{-1}\left\langle t_{w} w^{-1}\right\rangle$ is central. The centralizer in $\hat{\Gamma}_{w} \times \Gamma$ of $1 \times N$ is $\hat{\Gamma}_{w} \times 1$, and if $w \notin N$ then no element of $p_{1}^{-1}\left\langle t_{w} w^{-1}\right\rangle$ lies in $\hat{\Gamma}_{w} \times 1$. Thus, in the case $w \notin N$, the centre of $C_{\hat{P}_{w}}(h)$ is cyclic (generated by $\left\langle a_{1}\right\rangle$ ).

In case (2) of the preceding lemma, if $Q$ is torsion-free then any root of $u_{2}$ must lie in $N$ and one can avoid the need to pass to a subgroup of finite index, i.e. $C_{\hat{P}_{w}}(h) \cong\left(\left(\hat{\Gamma}_{w} \times 1\right) \cap \hat{P}_{w}\right) \times \mathbb{Z}$.

The following lemma shows that if $Q=\Gamma / N$ is infinite (in the construction of Theorem 3.1), then the centralizer in $\hat{P}_{w}$ of any non-trivial element of the form $\left(1, u_{2}\right)$ is not finitely generated (Case (2) above).

Lemma 4.6. Let $\Sigma$ be a set of coset representatives for $N$ in $\Gamma$ obtained by choosing (non-constructively) a word in $\underline{x}$ that represents each element of $Q=$ $\Gamma / N$. (The empty word is chosen to represent 1.) Then $\left(\hat{\Gamma}_{w} \times 1\right) \cap \hat{P}_{w}$ can be presented as the following $H N N$-extension of $N$ :

$$
\left\langle N, s_{\sigma}(\sigma \in \Sigma) \mid s_{\sigma}^{-1} \phi_{\sigma}\left(a_{1}\right) s_{\sigma}=\phi_{\sigma}\left(\phi_{w}\left(a_{1}\right)\right),(\sigma \in \Sigma)\right\rangle .
$$

In particular, since $Q$ is infinite, $\left(\hat{\Gamma}_{w} \times 1\right) \cap \hat{P}_{w}$ is not finitely generated (indeed its first homology is not finitely generated).

Proof. Let $\tau=\left(t_{w}, 1\right)$. We shall show that the map $\Theta$ sending $N$ to $N \times 1$ and $s_{\sigma}$ to $\sigma(\underline{X}) \tau \sigma(\underline{X})^{-1}$ is an isomorphism onto $\left(\hat{\Gamma}_{w} \times 1\right) \cap \hat{P}_{w}$.

First, in order to show that $\Theta$ is surjective, we shall express a typical element $v$ of $\hat{P}_{w}$ in a convenient form. Let $v=v_{0} \tau^{\varepsilon_{1}} v_{1} \cdots \tau^{\varepsilon_{n}} v_{n}$ where $v_{i} \in P$ is a word in the generators of $\hat{P}_{w}$ which is $\tau$-reduced. Using the relations of $P$ we can express $v_{0}$ in the form $v_{0}=u_{0} \sigma_{0}(\underline{X})$ where $\sigma_{0} \in \Sigma$ and $u_{0}$ is a word in $\underline{a}^{L} \cup \underline{a}^{R}$. Thus $v=u_{0} \sigma_{0} \tau^{\varepsilon_{1}} \sigma_{0}^{-1} \sigma_{0} v_{1} \cdots \tau^{\varepsilon_{n}} v_{n}$. Next express $\sigma_{0} v_{1}$ in the form $\sigma_{0} v_{1}=u_{1} \sigma_{1}(\underline{X})$ where $\sigma_{1} \in \Sigma$ and $u_{1}$ is a word in $\underline{a}^{L} \cup \underline{a}^{R}$. Continuing in this way we obtain

$$
v=u_{0} \sigma_{0} \tau^{\varepsilon_{1}} \sigma_{0}^{-1} u_{1} \cdots \sigma_{n-1} \tau^{\varepsilon_{n}} \sigma_{n-1}^{-1} u_{n} \sigma_{n}
$$

where $\sigma_{i} \in \Sigma$ and each $u_{i}$ is a word in $\underline{a}^{L} \cup \underline{a}^{R}$. Setting $s_{\sigma}=\sigma(\underline{X}) \tau \sigma(\underline{X})^{-1}$, this can be written as $v=u_{0} s_{\sigma_{0}}^{\varepsilon_{1}} u_{1} \cdots s_{\sigma_{n-1}}^{\varepsilon_{n}} u_{n} \sigma_{n}$.

Next observe that $s_{\sigma}=\left(\sigma(\underline{x}) t_{w} \sigma(\underline{x})^{-1}, 1\right)$ and so $s_{\sigma}$ commutes with any word in $\underline{a}^{R}$. Hence we can move all of the $\underline{a}^{R}$ symbols to the right and thus express $v$ in the form

$$
v=u_{0}^{L} \sigma_{0} \tau^{\varepsilon_{1}} \sigma_{0}^{-1} u_{1}^{L} \cdots \sigma_{n-1} \tau^{\varepsilon_{n}} \sigma_{n-1}^{-1} u_{n}^{L} u^{R} \sigma_{n}
$$

where the $u_{i}^{L}$ are words in $\underline{a}^{L}$ and $u^{R}$ is a word in $\underline{a}^{R}$ and $\sigma_{n}=\sigma_{n}(\underline{X})$.

Now, if $v \in \hat{\Gamma}_{w} \times 1$ then $u^{R} \sigma_{n}\left(\underline{x}^{R}\right)=1$ in $\Gamma$. But $u^{R} \in 1 \times N$, therefore $\sigma_{n}(\underline{x}) \in N$ and since $\sigma_{n}$ is one of the chosen coset representatives $\sigma_{n}$ is the empty 
word and $u^{R}=1$ in $\Gamma$. Thus $\left(\hat{\Gamma}_{w} \times 1\right) \cap \hat{P}_{w}$ is generated by the $\underline{a}^{L}$ together with the $s_{\sigma}(\sigma \in \Sigma)$ and so $\Theta$ is surjective.

It remains to show that $\Theta$ is injective. Let $v=u_{0}(\underline{a}) s_{\sigma_{1}}^{\varepsilon_{1}} u_{1}(\underline{a}) \cdots s_{\sigma_{n}}^{\varepsilon_{n}} u_{n}(\underline{a})$ be an element of $\operatorname{ker} \Theta$. It suffices to show that $v$ contains an " $s_{\sigma_{i}}-$ pinch". The image of this word in $\hat{\Gamma}_{w}=\hat{\Gamma}_{w} \times 1$ (dropping the superscript $L$ ) is: $v=$ $u_{0} \sigma_{1} \tau_{w}^{\varepsilon_{1}} \sigma_{1}^{-1} u_{1} \cdots \sigma_{n} \tau_{w}^{\varepsilon_{n}} \sigma_{n}^{-1} u_{n}$.

Applying Britton's Lemma to $\hat{G}_{w}$, we see that there exists a $t_{w}$-pinch, that is, for some $i$ there is a subword $t_{w}^{\varepsilon_{i}} \sigma_{i-1}^{-1} u_{i} \sigma_{i} t_{w}^{-\varepsilon_{i}}$ where either $\varepsilon_{i}=-1$ and $\sigma_{i-1}^{-1} u_{i} \sigma_{i}=$

$a_{1}^{m}$ in $\Gamma$, or $\varepsilon_{i}=+1$ and $\sigma_{i-1}^{-1} u_{i} \sigma_{i}=\phi_{w}\left(a_{1}\right)^{m}$ in $\Gamma$. In either case we must have $\sigma_{i-1}=\sigma_{i}$ since $N$ is normal in $\Gamma$ and $\sigma_{j} \in \Sigma$ are the chosen representatives. Thus $s_{\sigma_{i}}=s_{\sigma_{i-1}}$ and $\varepsilon_{i}=-\varepsilon_{i-1}$ and we have an $s_{\sigma_{i}}$ pinch in $v$.

The proof of Theorem 4.3. When $w=1$ in $Q$ (i.e. $w \in N$ ), we proved in Case 3 (b) of Lemma 4.5 that $\mathbb{Z}^{2} \times N$ arises as the centralizer of some element in $\hat{P}_{w}$. We claim that if $w \neq 1$ in $Q$, then $C_{1}:=\mathbb{Z}^{2} \times N$ is not isomorphic to the centralizer of any element of $\hat{P}_{w}$.

We refer to the numbering in Lemma 4.5. Since $N$ is not abelian, $C_{1}$ cannot arise in Case (1). Since $N$ is finitely generated, $C_{1}$ cannot arise in Case (2). Since abelian subgroups of $\Gamma$ are cyclic, $\mathbb{Z} \times \Gamma$ does not contain $\mathbb{Z}^{3}$ whereas $C_{1}$ does; this eliminates Case 3(a). Finally, the two cases in 3(b) are distinguished by the rank of the centres.

\section{Normalizers of subgroups}

It is now well-known that the centralizer of a finitely generated subgroup of a bi-automatic group $G$ is a rational subgroup, and given a finite set of elements of $G$, the membership problem for their centralizer is recursively solvable (see for instance [11]).

We show now that the same is not true for normalizers. Let $P \subset \Gamma \times \Gamma$ be the fibre product associated to $\Gamma \rightarrow Q$. First notice that:

Lemma 5.1. The normalizer of $P$ in $\Gamma \times \Gamma$ consists of the preimage of the centre of $Q$, i.e. $N_{\Gamma \times \Gamma}(P)=\left\{\left(n_{1}, n_{2}\right) \mid n_{1} n_{2}^{-1} \in Z(Q)\right\}$. In particular, $P$ is its own normalizer if and only if the centre of $Q$ is trivial.

Proof. Let $\left(g_{1}, g_{2}\right) \in \Gamma \times \Gamma$. Then, for all $\left(p_{1}, p_{2}\right) \in P$, we have

$$
\begin{aligned}
\left(g_{1}, g_{2}\right)^{-1}\left(p_{1}, p_{2}\right)\left(g_{1}, g_{2}\right) \in P & \Leftrightarrow\left(g_{1}^{-1} p_{1} g_{1}, g_{2}^{-1} p_{2} g_{2}\right) \in P \\
& \Leftrightarrow g_{1}^{-1} p_{1} g_{1} g_{2}^{-1} p_{2}^{-1} g_{2}=1 \text { in } Q \\
& \Leftrightarrow g_{2} g_{1}^{-1} p_{1} g_{1} g_{2}^{-1}=p_{2} \text { in } Q \\
& \Leftrightarrow g_{1} g_{2}^{-1} \in C_{Q}\left(p_{1}\right)
\end{aligned}
$$


since $p_{1}=p_{2}$ in $Q$. As $(x, x) \in P$ for each generator $x$ of $Q$, it follows that $g_{1} g_{2}^{-1} \in Z(Q)$, the centre of $Q$, if and only if $\left(g_{1}, g_{2}\right) \in N_{\Gamma \times \Gamma}(P)$.

Let $Q=\langle X \mid R\rangle$ be a group with an unsolvable word problem, and suppose that the presentation is aspherical. We may assume the group $Q$ has a trivial centre. (The group constructed in [9] actually has trivial centre, but we could also just replace $Q$ by its free product with an infinite cycle.) The corresponding fibre product $P$ constructed in Theorem A then has unsolvable membership problem and coincides with its normalizer. Thus:

Corollary 5.2. There exists a torsion-free word hyperbolic group $\Gamma$ and a finitely presented subgroup $P \subset \Gamma \times \Gamma$ such that membership in the normalizer $N_{\Gamma \times \Gamma}(P)$ is recursively undecidable.

For each $w \in F(X)$, let $U_{w}$ denote the group $U_{w}=(Q \times \mathbb{Z}) *_{\mathbb{Z}}(\mathbb{Z} \times \mathbb{Z})$, where the generator of the cyclic amalgamated subgroup is identified with $(w, 1)$ on the left, and with $(0,1)$ on the right. The centre of $Q \times \mathbb{Z}$ is the infinite cycle generated by $(1,1)$, where $Q$ is written multiplicatively and $\mathbb{Z}$ is written additively (with identity 0 ). The centre of the amalgamated free product $U_{w}$ is the intersection of the centres of the factors with the amalgamated subgroup. Thus

Lemma 5.3. The centre of $U_{w}$ is non-trivial if and only if $w=1$ in $Q$.

Since the presentation for $Q=\langle X \mid R\rangle$ is aspherical, it follows that $Q \times \mathbb{Z}$ is an $F_{3}$ group, indeed one obtains an Eilenberg-Maclane space for it by forming the product of a circle with the standard 2-complex for $\langle X \mid R\rangle$. The usual presentation for $\mathbb{Z} \times \mathbb{Z}$ is aspherical and $U_{w}$ is formed by amalgamating an infinite cyclic subgroup, so $U_{w}$ is also an $F_{3}$ group.

Let $1 \rightarrow N_{w} \rightarrow \Gamma_{w} \rightarrow U_{w} \rightarrow 1$ be the exact sequence constructed as in 3.1. Let $V_{w}$ be the associated fibre product. From 5.1 and 5.3 we have:

Corollary 5.4. The normalizer of $V_{w}$ in $\Gamma_{w} \times \Gamma_{w}$ is equal to $V_{w}$ if and only if $w \neq 1$ in $Q$.

By the 1-2-3 Theorem, each $V_{w}$ is finitely presented and we can explicitly write down a presentation. It follows that:

Theorem 5.5. There is an explicit, recursive class of finite presentations of biautomatic groups $\Gamma_{w} \times \Gamma_{w}$ together with finite presentations of subgroups $V_{w}$ and explicit embeddings $V_{w} \rightarrow \Gamma_{w} \times \Gamma_{w}$ such that there is no algorithm to decide whether or not the normalizer of $V_{w}$ in $\Gamma_{w} \times \Gamma_{w}$ is equal to $V_{w}$. 


\section{References}

[1] J. Alonso and M. R. Bridson, Semihyperbolic groups, Proc. Lon. Math. Soc. 70 (1993), 56-114.

[2] Y. G. Baik, J. Harlander and S. J. Pride, The geometry of group extensions, J. Group Theory 1 (1998), 395-416.

[3] G. Baumslag, M. R. Bridson, C. F. Miller III and H. Short, Finitely presented subgroups of automatic groups and their isoperimetric functions, Journ. Lon. Math. Soc. 56 (1997), 292-304.

[4] G. Baumslag, C. F. Miller III and H. Short, Unsolvable problems about Small Cancellation and Word Hyperbolic groups, Bull. Lon. Math. Soc. 25, (1993), 97-101.

[5] G. Baumslag and J. E. Roseblade, Subgroups of direct products of free groups, Journ. Lon. Math. Soc. 30 (1984), 44-52.

[6] M. R. Bridson and A. Haefliger, "Metric spaces of non-positive curvature", Grundlehren der Mathematischen Wiss., Vol. 319, Springer-Verlag, Heidelberg, 1999.

[7] I. M. Chiswell, D. J. Collins and J. Huebschmann, Aspherical group presentations, Math. Zeit. 178 (1981), 1-36.

[8] D. J. Collins and C. F. Miller III, The Conjugacy problem and subgroups of finite index, Proc. Lon. Math. Soc. 33 (1977), 535-556.

[9] D. J. Collins and C. F. Miller III, The word problem in groups of cohomological dimension 2. In: Groups St. Andrews in Bath, LMS Lecture Notes 270 (1998), (eds. Campbell, Robertson, Smith), pp. 211-218.

[10] D. B. A. Epstein, with J. W. Cannon, D. F. Holt, S. V. F. Levy, M. S. Paterson and W. P. Thurston, Word processing in Groups, Jones and Barlett, Boston-London 1992.

[11] S. M. Gersten and H. Short, Rational subgroups of biautomatic groups, Annals of Math 134 (1991), 125-158.

[12] S. Gersten and H. Short, Small cancellation theory and automatic groups, Invent. Math. 102 (1990), 305-334.

[13] M. Gromov, Hyperbolic groups. In: Essays on group theory, MSRI Publications No. 8, edited by S.M. Gersten, Springer-Verlag, 1987.

[14] F. J. Grunewald, On some groups which cannot be finitely presented, Journ. Lon. Math. Soc. 17 (1978), 427-436.

[15] R. C. Lyndon and P. E. Schupp, Combinatorial group theory, Springer-Verlag, BerlinHeidelberg-New York 1977.

[16] G. A. Niblo and L. D. Reeves, The geometry of cube complexes and the complexity of their fundamental groups, Geometry and Topology 1 (1997), 1-7.

[17] C. F. Miller III, On group-theoretic decision problems and their classification, Annals of Mathematics Studies, No. 68, Princeton University Press (1971).

[18] C. F. Miller III, Decision problems for groups: survey and reflections. In: Algorithms and Classification in Combinatorial Group Theory (eds. G Baumslag and C. F. Miller III), MSRI Publications No. 23, Springer-Verlag 1992, pp. 1-59.

[19] S. J. Pride, Identities among relations of group presentations. In: Group Theory from a Geometrical Viewpoint, edited by E. Ghys, A. Haefliger and A. Verjovsky, World Scientific 1991, pp. 687-717.

[20] E. Rips, Subgroups of small cancellation groups, Bull. Lon. Math. Soc. 14 (1982), 45-47.

[21] M. Shapiro, Automatic structure and graphs of groups. In: Topology '90, edited by B. Apanasov et al., de Gruyter, 1991.

[22] H. Short, Groups and Combings, École Normale Supérieure de Lyon, preprint \# 29 (1990).

[23] D. Wise, Incoherent negatively curved groups, Proc. Amer. Math. Soc. 126 (1998), 957-964. 
Gilbert Baumslag

Department of Mathematics

City College of New York

Convent Avenue at 138th Street

New York, NY 10031

USA

e-mail: gilbert@groups.sci.ccny.cuny.edu

Martin R. Bridson

Mathematical Institute

24-29 St. Giles

Oxford OX1 3LB

U.K.

e-mail: bridson@maths.ox.ac.uk

(Received: October 7, 1999)
Charles F. Miller III

Department of Mathematics and Statistics

University of Melbourne

Parkville 3052

Australia

e-mail: cfm@ms.unimelb.edu.au

Hamish Short

U.R.A. 225

Centre de Mathématiques et d'Informatique

Rue Joliot-Curie

Université de Provence

F-13453 Marseille cedex 13

France

e-mail: hamish@claudia.univ-mrs.fr 